\title{
Abalone visceral extract inhibit tumor growth and metastasis by modulating Cox-2 levels and CD8+ T cell activity
}

\author{
Choong-Gu Lee', Ho-Keun Kwon', Jae Ha Ryu', Sung Jin Kang ${ }^{1}$, Chang-Rok Im², Jae II Kim', Sin-Hyeog Im ${ }^{1 *}$
}

\begin{abstract}
Background: Abalone has long been used as a valuable food source in East Asian countries. Although the nutritional importance of abalone has been reported through in vitro and in vivo studies, there is little evidence about the potential anti-tumor effects of abalone visceral extract. The aim of the present study is to examine antitumor efficacy of abalone visceral extract and to elucidate its working mechanism.

Methods: In the present study, we used breast cancer model using BALB/c mouse-derived 4T1 mammary carcinoma and investigated the effect of abalone visceral extract on tumor development. Inhibitory effect against tumor metastasis was assessed by histopathology of lungs. Cox-2 productions by primary and secondary tumor were measured by real-time RT-PCR and immunoblotting (IB). Proliferation assay based on $\left.{ }^{3} \mathrm{H}\right]$-thymidine incorporation and measurement of cytokines and effector molecules by RT-PCR were used to confirm tumor suppression efficacy of abalone visceral extract by modulating cytolytic CD8+ T cells. The cytotoxicity of $C D 8^{+} T$ cell was compared by JAM test.

Results: Oral administration of abalone visceral extract reduced tumor growth (tumor volume and weight) and showed reduced metastasis as confirmed by decreased level of splenomegaly (spleen size and weight) and histological analysis of the lung metastasis (gross analysis and histological staining). Reduced expression of Cox-2 (mRNA and protein) from primary tumor and metastasized lung was also detected. In addition, treatment of abalone visceral extract increased anti-tumor activities of $\mathrm{CD}^{+} \mathrm{T}$ cells by increasing the proliferation capacity and their cytolytic activity.
\end{abstract}

Conclusions: Our results suggest that abalone visceral extract has anti-tumor effects by suppressing tumor growth and lung metastasis through decreasing Cox-2 expression level as well as promoting proliferation and cytolytic function of $\mathrm{CD} 8^{+} \mathrm{T}$ cells.

\section{Background}

Abalones are medium to very large-sized edible sea snails, marine gastropod mollusks in the family Haliotidae and the genus Haliotis [1]. Abalones are largely cultivated and used as valuable food resources in East Asian countries. In Korea, not only the protein-rich body part of abalone but also the viscera portion is taken in the form of sashimi or pickle and used as stamina food from ancient times. Although the nutritional

\footnotetext{
* Correspondence: imsh@gist.ac.kr

'School of Life Sciences and Immune Synapse Research Center, Gwangju Institute of Science and Technology (GIST), Gwangju 500-712, Republic of Korea

Full list of author information is available at the end of the article
}

composition of abalone visceral extract have not yet been identified in depth, since abalone live on brown algae like Ecklonia, Laminaria and Undaria for cultivation [2], it is regarded that the visceral portion of abalone may contain concentrated nutritional components derived from sea weed. Besides, it is well known that polysaccharides and glycoproteins of the brown algae possess potential immune-stimulant, anti-tumoral and anti-viral activity [3]. However, there is still not much information about the nutritional effects of abalone viscera itself.

There are some previous reports that the body and visceral portion of Haliotis discus hannai showed in vitro antioxidant activity $[4,5]$. Antioxidants are 
present in abundance in the dietary substances and their potent chemopreventive effects against many type of cancers have been reported [6]. The water extract of abalone has anti-tumor effects [7]. However, mechanisms involved in the anti-tumor effects of extract from abalone viscera have not been fully clarified. The purpose of this study is to evaluate the anti-tumor activity of abalone visceral extract in vivo using mouse breast cancer as a model and to elucidate underlying mechanisms involved in this protective effect.

Breast cancer is one of the major type of cancer affecting women and ranks second in causing death in women [8]. Although many clinical trials and basic research have been performed to subdue this disease, there is no conclusive treatment or medicine. Moreover, current conventional chemotherapies have severe side effects and toxicity $[9,10]$. Hence, there are increasing numbers of studies to prevent cancers using dietary factors $[11,12]$. To evaluate the anti-tumor effects of abalone visceral extract on breast cancer model, we used $4 \mathrm{~T} 1$ murine mammary carcinoma cells that have highly metastatic characteristics at an early stage and mimic the human breast cancer $[13,14]$. Oral administration of abalone visceral extract significantly inhibited tumor progression by decreasing the levels of Cox-2, EGF, VEGF, and FGF in primary tumor as well as metastatic lesions. In addition, abalone visceral extract potentiated proliferation and cytolytic activity of $\mathrm{CD}^{+} \mathrm{T}$ cells.

\section{Methods}

\section{Animals}

Balb/c mice were purchased from SLC (Hamamatsu, Japan) and maintained under specific pathogen-free conditions in the animal facility of the Gwangju Institute of Science and Technology. Animal experiments were performed in accordance with protocols approved by the animal care and use committees of the Gwangju Institute of Science and Technology.

\section{Preparation of the abalone visceral extract}

Visceral portion of Haliotis discus hannai were obtained from live abalone. For $500 \mathrm{~g}$ of abalone visceral mass, we added 2 liters of $30 \%$ acetic acid then homogenized thoroughly. Then, the mixture was incubated at $4{ }^{\circ} \mathrm{C}$ for 12 hours with rotation. Next, the mixture was centrifuged at $4^{\circ} \mathrm{C}$ for 1 hour with 5,000 rpm. Supernatant fraction was harvested and completely freeze-dried for 72 hours by using a freeze-dryer (Ilshin, Korea). The dried powder extract was further finely grounded by mortar and pestle and solubilized in sterilized PBS at 50 $\mathrm{mg} / \mathrm{ml}$ concentration. Reverse phase high-performance liquid chromatography (RP-HPLC) assay was performed and relative amounts laminarin and D-mannitol were used as standard makers for the quality control of abalone visceral extract composition in each experiment (Additional file 1: Figure S1).

\section{Tumor induction and anti-tumor assay}

Induction of breast cancer was carried out as described [15] with minor modifications. Mice were divided into two groups of abalone visceral extract-fed versus control PBSfed. Sex- and age-matched mice with similar body weight were fed with either abalone visceral extract ( $5 \mathrm{mg} /$ dose) or control PBS every day for 2 weeks prior to tumor transplantation. $1 \times 10^{6}$ cells $/ 0.1 \mathrm{ml}$ of $4 \mathrm{~T} 1$ mouse mammary carcinoma cells were injected into a subcutaneous space on the flank of each mouse (female Balb/c mice from 6 to 8 weeks). Tumor size was measured with a Vernier caliper every 2 days from post-tumor induction, and tumor volumes were measured by the standard formula: width ${ }^{2} \times$ length $\times 0.52$. Mice were sacrificed for further analysis at the 25 days from post-tumor induction.

\section{Cell isolation and culture}

$\mathrm{CD}^{+} \mathrm{T}$ cells were purified from the draining lymph nodes using $\mathrm{CD}^{+} \mathrm{T}$ cell isolation kit (Miltenyi Biotech, Germany). For the activation, $\mathrm{CD}^{+} \mathrm{T}$ cells were stimulated with $10 \mu \mathrm{g} / \mathrm{ml}$ plate-bound anti-CD3, $10 \mu \mathrm{g} / \mathrm{ml}$ soluble anti-CD28 in RPMI 1640 medium (Welgene, Korea) supplemented with $10 \%$ fetal bovine serum, Lglutamine, penicillin-streptomycin, nonessential amino acids, sodium pyruvate, vitamins, HEPES and $\beta$-mercaptoethanol. Anti-CD3 (145.2C11) and anti-CD28 (37.51) were purchased from BD Biosciences.

\section{Histopathology}

Lung tissues of mice in each group were dissected and fixed in $4 \%$ formaldehyde. After embedding in paraffin, the tissue sections $(5 \mu \mathrm{m})$ were mounted on glass slides and stained with hematoxylin and eosin following the previous report [15]. The specimens were observed under a light microscope (Olympus, Germany).

\section{RNA Isolation, cDNA synthesis, quantitative RT-PCR}

Total RNA was extracted from the cells using TRIzol reagent (Molecular Research Center, USA) according to the manufacturer's protocol. For reverse transcription, cDNA was generated using $1 \mu \mathrm{g}$ of total RNA, oligo(dT) primer (Promega) and Improm-II Reverse Transcriptase (Promega) in a total volume of $20 \mu \mathrm{l}$. One microliter of cDNA was amplified using the specific primers (Table. 1). Mouse hypoxanthine-guanine phosphoribosyl transferase (HPRT) primer was used for quantitative RT-PCR to normalize the amount of cDNA used for each condition.

\section{Proliferation assay}

Assessment of lymphocyte responses against antiCD3 and anti-CD28 stimulation was determined by 


\begin{tabular}{|c|c|c|}
\hline Genes & Sense & Antisense \\
\hline HPRT & TTATGGACAGGACTGAAAGAC & GCTITAATGTAATCCAGCAGGT \\
\hline Cox-2 & TGAGCAACTATTCCAAACCAGC & GCACGTAGTCTTCGATCACTATC \\
\hline VEGF & GCACATAGAGAGAATGAGCTTCC & CTCCGCTCTGAACAAGGCT \\
\hline FGF & ACCCACACGTCAAACTACAAC & CACTCCCTTGATAGACACAACTC \\
\hline EGF & TTCTCACAAGGAAAGAGCATCTC & CTGCTGTCCCGTTAAGGAAAAC \\
\hline MMP13 & CTTCTTCTTGTTGAGCTGGACTC & CTGTGGAGGTCACTGTAGACT \\
\hline GzmB & CCACTCTCGACCCTACATGG & GGCCCCCAAAGTGACATTTATT \\
\hline GzmC & GCAGAGGAGATAATCGGAGGC & GCACGAATTTGTCTCGAACCA \\
\hline FasL & TCCGTGAGTTCACCAACCAAA & GGGGGTTCCCTGTTAAATGGG \\
\hline TNF- $\alpha$ & CATCTTCTCAAAATTCGAGTGACAA & TGGGAGTAGACAAGGTACAACCC \\
\hline $\mathrm{IFN}-\gamma$ & GAGCCAGATTATCTCTITCTACC & GTTGTTGACCTCAAACTTGG \\
\hline
\end{tabular}

$\left[{ }^{3} \mathrm{H}\right]$-thymidine incorporation assays. Briefly, isolated $\mathrm{CD}^{+}$lymphocytes were plated in flat-bottom 96-well plates and cultured in the presence or absence of stimulation. After 56 hours of incubation, $0.5 \mu \mathrm{Ci}$ of $\left[{ }^{3} \mathrm{H}\right]$-thymidine (NEN, USA) was added, and cells were pulse labeled for $16 \mathrm{~h}$. The degree of proliferation was presented as counts per minutes detected by scintillation counter (Beckman, USA).

\section{Total lysate preparation and immunoblotting}

For total lysate preparation, tumor and lung tissues were first homogenized using homogenizer (Pro Scientific, USA) and then centrifuged briefly. Next, pelleted cells were lysed in RIPA buffer ( $50 \mathrm{mM}$ Tris, $\mathrm{pH}$ 7.6, 150 $\mathrm{mM} \mathrm{NaCl}, 1 \% \mathrm{NP}-40)$ containing protease inhibitor cocktails (Roche, USA) for $10 \mathrm{~min}$ on ice. To determine levels of Cox-2 proteins, total lysates from each sample were prepared described above and loaded together with a PageRuler Prestained Protein ladder (Fermentas, Canada) on a $10 \%$ SDS-PAGE gel. The proteins were electroblotted onto a nitrocellulose membrane (Bio-Rad, USA). After blocking, the membranes were incubated with a 1/500 dilution of anti-Cox-2 (Cayman chemical, USA) in blocking buffer overnight at $4^{\circ} \mathrm{C}$. The blots were developed using a 1/10,000 diluted anti-rabbit HRP (Abcam, USA) and visualized by ECL solution (Amersham, UK). To confirm the amount of sample loading and transfer, membranes were incubated in stripping buffer, re-blocked for $1 \mathrm{~h}$, and reprobed with anti- $\beta$-tubulin (Santa Cruz) with anti-mouse HRP (Abcam).

\section{JAM test}

The cytotoxicity of $\mathrm{CD}^{+} \mathrm{T}$ cell was compared by JAM test with minor modification [16]. EL4 mouse lymphoma cells were used as target cells. Target cells were labeled with $5 \mu \mathrm{Ci} / \mathrm{ml}$ of $\left[{ }^{3} \mathrm{H}\right]$-thymidine (NEN, USA) for 12 hours at $37^{\circ} \mathrm{C}$. Labeled target cells were harvested, washed 3 times with PBS, and seeded in 96-well flat-buttom plates at a density of $1 \times 10^{4}$ cells/well. $\mathrm{CD} 8^{+} \mathrm{T}$ cells were used as effector cells. Activated $\mathrm{CD} 8^{+} \mathrm{T}$ cells were washed and added in various ratios to the target cells. After 16 hours co-culture, remained radioactivity from intact cells can be trapped in the filter paper and then the filter discs were measured on a microplate beta counter (Beckman, USA). Data were expressed as the percentage of cytotoxicity calculated by the following formula: [(cpm spontaneous - cpm experiment)/cpm spontaneous] $\times 100$.

\section{Statistical analysis}

All the data were expressed as mean \pm standard error $(\mathrm{SE})$. For statistical analysis, two-tailed student's $t$-test was employed unless otherwise stated. Differences were considered statistically significant with a $p$-value of $\leq 0.05(*<0.05, * * 0.01, * * *<0.001)$.

\section{Results}

\section{Administration of abalone visceral extract suppresses tumor growth}

To ensure the quality and purity of each preparation of abalone visceral extract, RP-HPLC analysis was performed by measuring the content of known active compounds such as laminarin and D-mannitol which are known as major polysaccharide constituents in Laminaria, the food for abalone. (Additional file 1: Figure S1).

To determine the dose of abalone visceral extract that does not induce in vivo toxicity upon oral administration, Balb/C mice were fed with $5 \mathrm{mg}$ of abalone visceral extract using feeding needle every other day for 20 days and the body weight was measured (Additional file 2: Figure S2A). Overall, mice viability and changes in body weight were not affected by administration of abalone visceral extract. In addition, gross analysis of the intestine did not show any signs of inflammation both in the control (PBS) and abalone visceral extract fed group (Additional file 2: Figure S2B). Anti-cancer effect of abalone visceral extract was tested in mouse breast cancer model in Balb/C as 

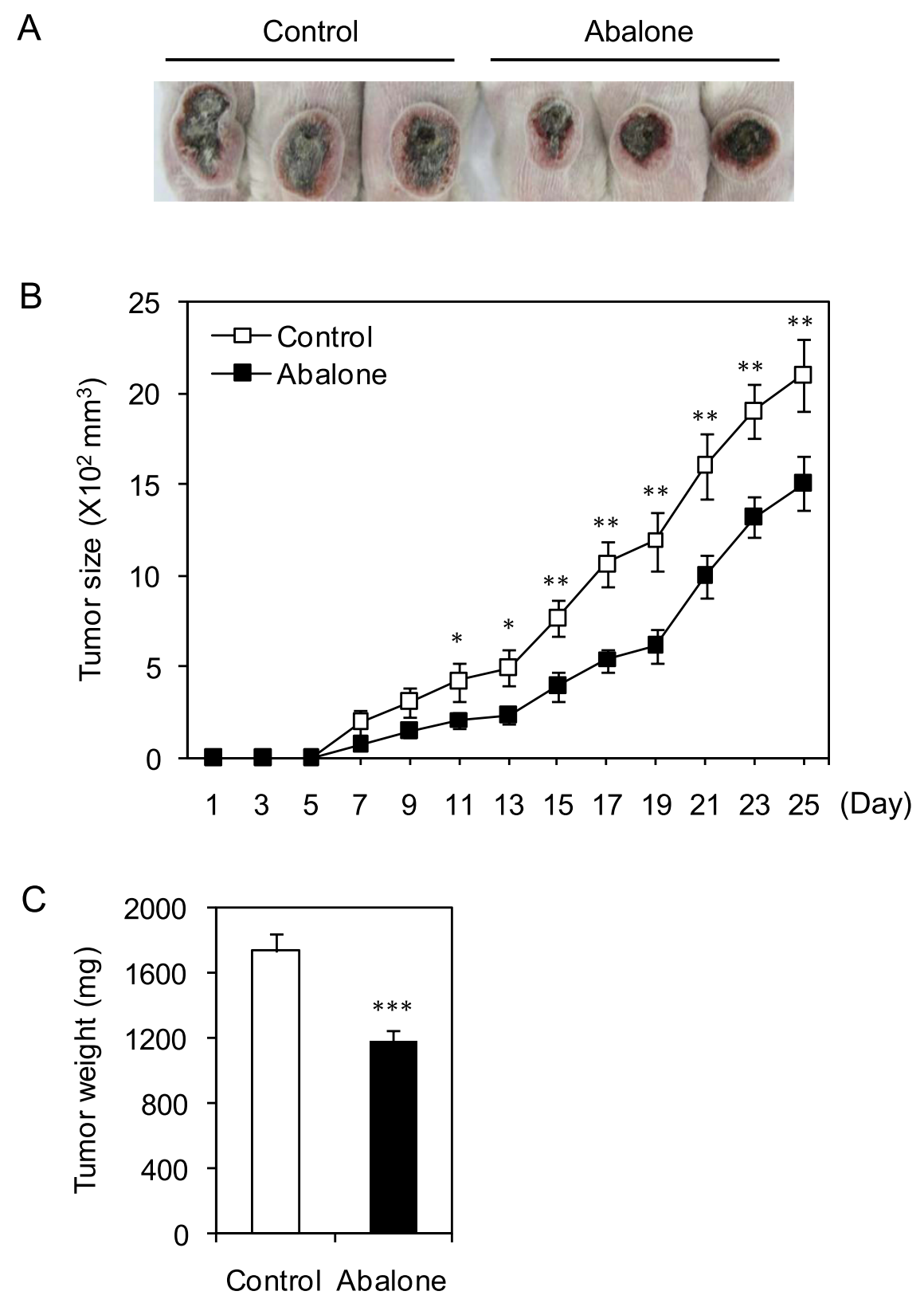

Figure 1 Suppression of tumor progression by administration of abalone visceral extract. Balb/c female mice were orally administered with abalone visceral extract (100 ul of $50 \mathrm{mg} / \mathrm{ml}$ ) for 2 weeks before cancer transplantation. Mouse breast cancer model was induced by injection of $4 \mathrm{~T} 1$ cell $\left(1 \times 10^{6}\right.$ cells) into flank backs. Abalone visceral extract or PBS as control was daily treated by oral administration for 25 days post tumor induction. (A) Picture of tumor from each group was taken. (B) During the treatment period, tumor size was measured every 2 days and shown in graph. (C) After 25 days from post-tumor induction, mice from each group were scarified and the weight of tumor mass was measured. Error bars indicated SE. Data are representative of five independent experiments. One $\left(^{*}\right)$, two $\left(^{* *}\right)$ or three asterisks $\left({ }^{* * *}\right)$ indicate $p<0.05, p<0.01$ or $p<0.001$, respectively.

described in Material and Method section. Mice were fed either with $5 \mathrm{mg}$ of the abalone visceral extract or PBS as a control for 2 weeks. And then breast cancer was induced by intradermal injection of $4 \mathrm{~T} 1$ mouse mammary carcinoma cells. Oral administration of abalone visceral extract continued for additional 25 days till the end of experiment. Administration of abalone visceral extract significantly decreased the size of tumor formation compared with control groups in overall. Administration of abalone visceral extract significantly (about 30\%) reduced tumor size 
compared with control group by gross analysis (Figure. 1A). During the treatment periods, tumor size was measured starting from day 7 of post-induction of cancer and till the end of experiments, 25 day of post-induction (Figure. 1B). In addition, administration of abalone visceral extract significantly reduced tumor mass compared with the control group (Figure. 1C). These data suggest that oral treatment of abalone visceral extract significantly reduced the development and progress of tumor formation in breast cancer model.

\section{Administration of abalone visceral extract inhibits tumor metastasis}

The $4 \mathrm{~T} 1$ breast cancer cells are one of the highly metastatic cancer cells [14] and metastatic splenomegaly is known as the first manifestation of metastasis in breast cancer $[17,18]$. Oral administration of the abalone visceral extract reduced the lymphomegaly (data not shown) and metastatic splenomegaly as evident from the decreased size (Figure. 2A) and weight of spleen (Figure. 2B). From these results, we hypothesized that abalone visceral extract could suppress the metastasis of the primary tumor. Since lung is the one of the predominant site of metastasis in breast cancer [19-21], we first compared the metastatic state of lungs between the experimental groups by gross examination (Figure. 2C). Control group showed deposited secondary tumors with various sizes (Figure. $2 \mathrm{C}$, middle panel). In contrast, the lung of abalone visceral extract fed group showed no secondary tumors (Figure. 2C, right panel). For more detailed examination of metastatic state of lungs from each group, histological examination was performed by
A

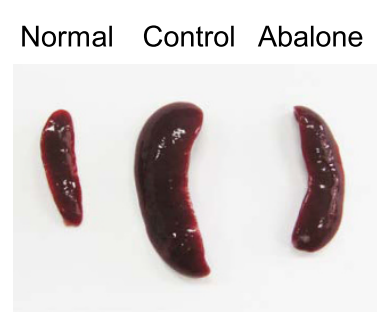

B

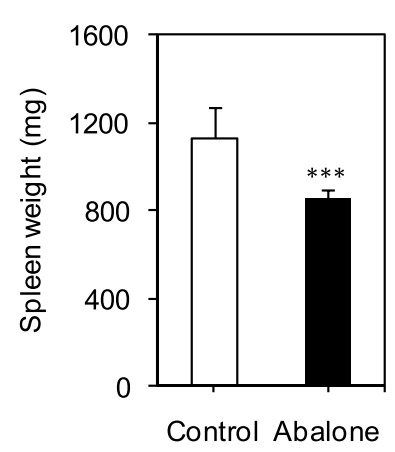

C

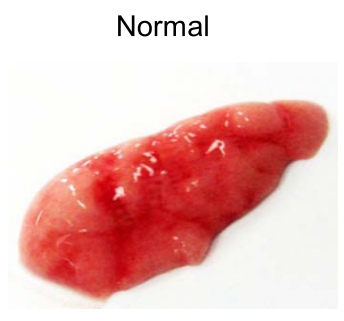

D



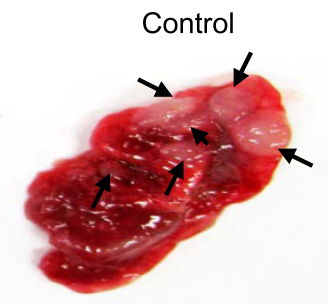

Control

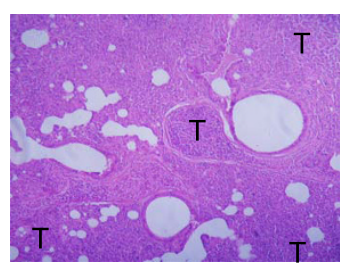

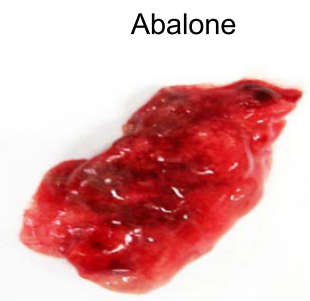

Abalone

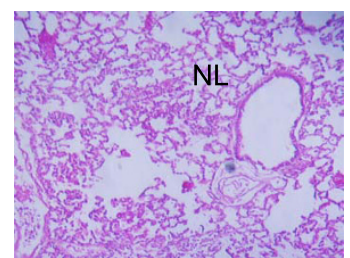

$(x 200)$

Figure 2 Effects of abalone visceral extract on tumor metastasis. (A) The gross analysis of spleen from normal healthy, fed with abalone visceral extract or PBS control treated group. (B) Spleen weight was measured and shown in graph. Values are means with $S E, n=5$. Data are representative of five independent experiments. One $\left({ }^{*}\right)$, two $\left(^{* *}\right)$ or three asterisks $\left({ }^{* * *}\right)$ indicate $p<0.05, p<0.01$ or $p<0.001$, respectively. (C) Gross appearance of lung from each treatment was shown and compared with normal healthy lung. Arrows from control lung picture indicate metastatic foci (middle panel). (D) H\&E-stained section of lung from each group was shown. Normal lung tissue is indicated by 'NL'. Tumor metastases are indicated by 'T'. Original magnifications; $\times 200$ 
hematoxylin and eosin (H\&E) staining (Figure. 2D). Unlikely the normal alveolar structure of normal healthy lungs (Figure. 2D, left panel), the control group showed massive infiltration of the tumors with increased numbers of small lymphatic or blood vessels (Figure. 2D, middle panel). Interestingly, lung from the abalone visceral extract treated group showed normal alveolar structure similar to the normal healthy group while control group showed destructed alveolar (Figure. 2D, right panel). These data indicate that treatment of abalone visceral extract inhibited metastatic development of breast cancer in lung tissues.

\section{Administration of abalone visceral extract suppress} primary tumor growth by decreasing Cox-2 expression Elevated Cox-2 expression is associated with increased tumor size during breast cancer progression [22], while specific knockdown of Cox-2 directly reduced level of $\mathrm{PGE}_{2}$ synthesis and tumor cell growth in 4T1 cells [23]. We investigated whether anti-cancer effect of abalone visceral extract (Figure. 1 and Figure. 2) is linked with modulation of Cox-2 expression level. Since Cox-2 is regulated at transcriptional and post-translational levels [24], Cox-2 transcript and protein levels were analyzed by real-time PCR and Western blot analysis, respectively from the tumor cells of breast cancer mice treated either with control (PBS) or abalone visceral extract. Mice fed with abalone visceral extract showed significantly reduced Cox-2 mRNA (Figure. 3A) and protein (Figure. 3B) levels compared to PBS fed control group. Tumorigenesis is often accompanied with angiogenic development [25]. Therefore, we further investigated whether oral administration of abalone visceral extract could also affect expression levels of VEGF, FGF and EGF. Indeed, abalone visceral extract treated group showed significantly reduced mRNA level of such targets molecules in the tumor tissues (Figure. 3C). These results suggest that the potent anti-tumor effect of abalone visceral extract is associated with down-regulation of Cox-2 expression level, as well as reduced transcript levels of tumor growth related angiogenic molecules such as VEGF, FGF and EGF in tumor tissues.

\section{Abalone visceral extract inhibits tumor metastasis by modulating Cox-2 expression}

Cox 2 plays important roles in breast cancer metastasis to bone [26] and increased Cox-2 level was identified as one of the markers for metastasis [27]. Therefore, we questioned whether suppression of tumor metastasis by oral administration of abalone visceral extract correlates with down-regulation of Cox-2 level in metastatic tissue. The mRNA and protein levels of Cox-2 were determined from the lung tissue isolated from each treatment group. Indeed, administration of abalone visceral extract



B

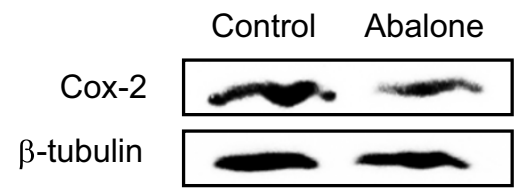

C

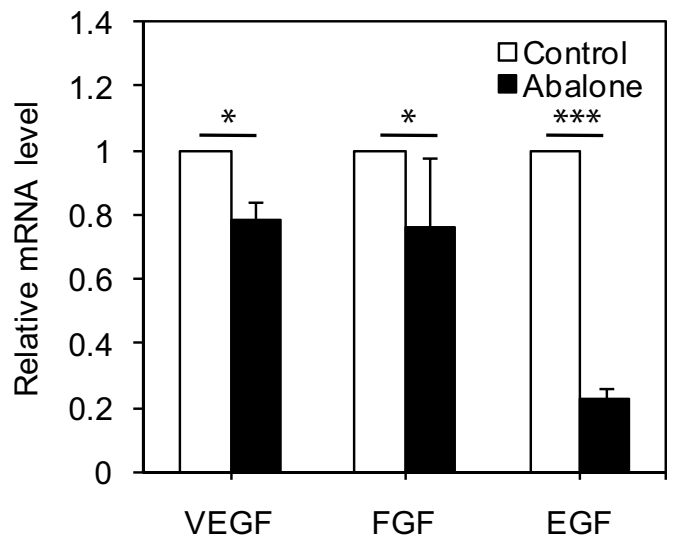

Figure 3 Effect of administration of abalone visceral extract on the levels of Cox-2 and angiogenic markers in primary tumor. (A) Primary tumor mass were homogenized and Cox-2 mRNA level was measured in each group. (B) Protein level of Cox-2 was detected using total cell lysates from each group. $\beta$-tubulin was used as loading control. (C) Angiogenic markers of tumor growth were measured by quantitative real-time PCR. Values are means with SE. Data are representative of five times independent experiments. One $\left(^{*}\right)$, two $\left(^{(*}\right)$ or three asterisks $\left({ }^{* *}\right)$ indicate $p<$ $0.05, p<0.01$ or $p<0.001$, respectively.

significantly reduced Cox-2 levels compared to PBS fed group both in mRNA (Figure. 4A) and protein (Figure. 4B) levels. To further check whether oral administration of abalone visceral extract also affected expression levels of metastasis related molecules, we analyzed the mRNA levels of VEGF, FGF and MMP-13 [28,29]. Indeed, administration of abalone visceral extract significantly decreased their expression levels (Figure. 4C). Taken 


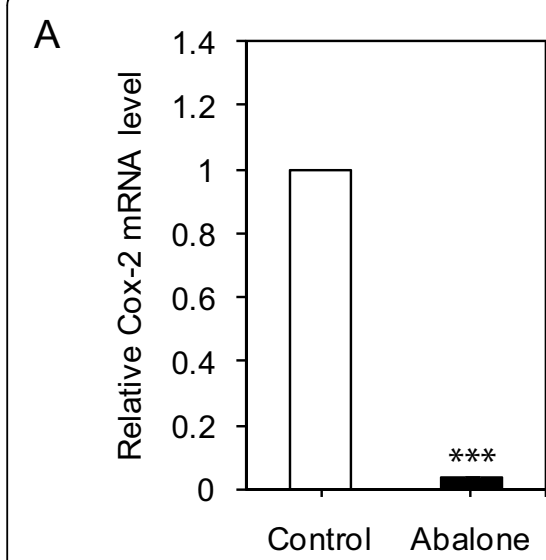

B

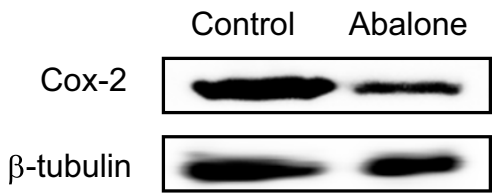

C

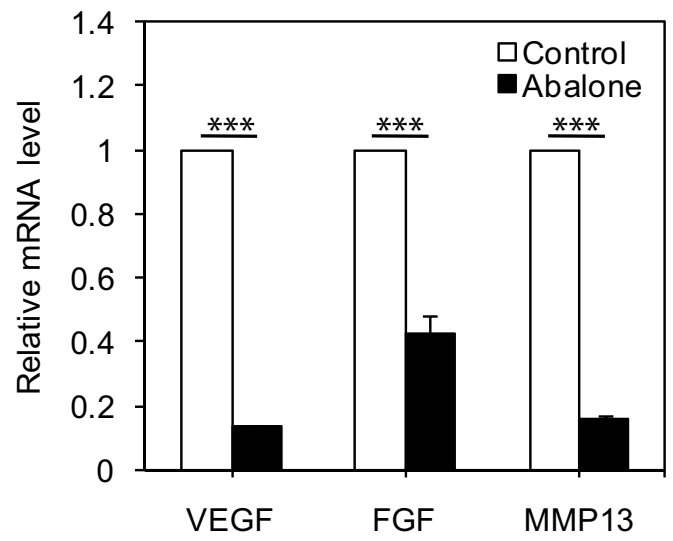

Figure 4 Abalone visceral extract inhibits tumor metastasis by modulating Cox-2 and Cox-2 related tumor metastasis markers. (A) Metastasized lung mass were homogenized and Cox-2 mRNA level was measured in each group by quantitative real-time PCR. (B) Protein level of Cox-2 was detected using total cell lysates from each group. $\beta$-tubulin was used as loading control. (C) Markers of tumor metastasis were measured by quantitative real-time PCR. Values are means with SE. Data are representative of five times independent experiments. One $\left(^{*}\right)$, two $\left(^{* *}\right)$ or three asterisks $\left({ }^{* *}\right)$ indicate $p<0.05, p<0.01$ or $p<0.001$, respectively.

together, these data suggest that the potent anti-metastatic effect of abalone visceral extract is associated with down-regulation of Cox-2 expression in metastatic lesions, as well as suppression of metastatic targets molecules such as VEGF, FGF and MMP-13.

\section{Abalone visceral extract potentiates the $\mathrm{CD}^{+}$cytotoxic} T cell activity

Chemopreventive approaches for tumor cells are often targeting modulation of tumor environment not only by exerting anti-tumor activity but also by regulating the immune system [30]. Among various types of immune cells, $\mathrm{CD}^{+}$cytotoxic $\mathrm{T}$ cells are well known for their anti-tumor activity by directly killing transformed cells through the perforin-granzyme pathway and death ligand such as Fas and tumor-necrosis factor [31]. We tested the possibility that the anti-tumor activity of abalone visceral extract may also modulate the functional activity of $\mathrm{CD}^{+} \mathrm{T}$ cells. Firstly, we performed $\mathrm{CD} 8^{+}$ $\mathrm{T}$ cell proliferation assay. Abalone visceral extract-fed group showed significantly increased $(\sim 30 \%)$ proliferation capacity compared with control $\mathrm{CD}^{+} \mathrm{T}$ cells from PBS-fed group (Figure. 5A). And then, the expression levels of cytokines related with effector function of CD8 $+\mathrm{T}$ cells were determined by quantitative RT-PCR. TNF- $\alpha$ and IFN- $\gamma$ are known important mediators of functionality of $\mathrm{CD}^{+} \mathrm{T}$ cells against tumor pathogenesis [32]. $\mathrm{CD}^{+} \mathrm{T}$ cells from the abalone visceral extract-fed groups showed significantly increased levels of IFN- $\gamma$ and TNF- $\alpha$ (Figure. 5B). Then, we compared the mRNA levels of genes related with cytolytic activity of $\mathrm{CD} 8^{+}$ $\mathrm{T}$ cells such as granzymes (gzmB and gzmC) and Fas ligand (FasL) (Figure. $5 \mathrm{C}$ ). $\mathrm{CD} 8^{+} \mathrm{T}$ cells from the extract-fed group showed increased mRNA level of the above target genes compared with $\mathrm{CD}^{+} \mathrm{T}$ cells from the control group. Since the expression level of cytolytic molecules is directly linked with the killing activity of $\mathrm{CD}^{+} \mathrm{T}$ cells [33], we tested the hypothesis that $\mathrm{CD} 8^{+}$ $\mathrm{T}$ cells from the abalone visceral extract-treated groups exhibited an increased CTL response than those from the control group. We performed the JAM test to test this hypothesis. Indeed, $\mathrm{CD}^{+} \mathrm{T}$ cells from the abalone visceral extract-treated groups showed significantly increased killing activity than that of the control group in a dose-dependent manner (Figure. 5D). These results indicate that abalone visceral extract potentiates the proliferative capacity and cytolytic activity of $\mathrm{CD} 8^{+}$ $\mathrm{T}$ cells in tumor draining lymph nodes.

\section{Discussion}

Abalone is often called as 'The emperor of the seashells' in Korea and mostly consumed as stamina food for sick and weak individuals because of its high content of proteins and vitamins [5]. Abalone mainly live on sea weeds that contain rich and concentrated nutritional components [2]. Apart from its known nutritional importance, there are not many studies on the effects of the abalone visceral extract. One of the known effects of abalone visceral extract is its antioxidant activity as demonstrated $[4,5]$. However, there are still few in vivo 


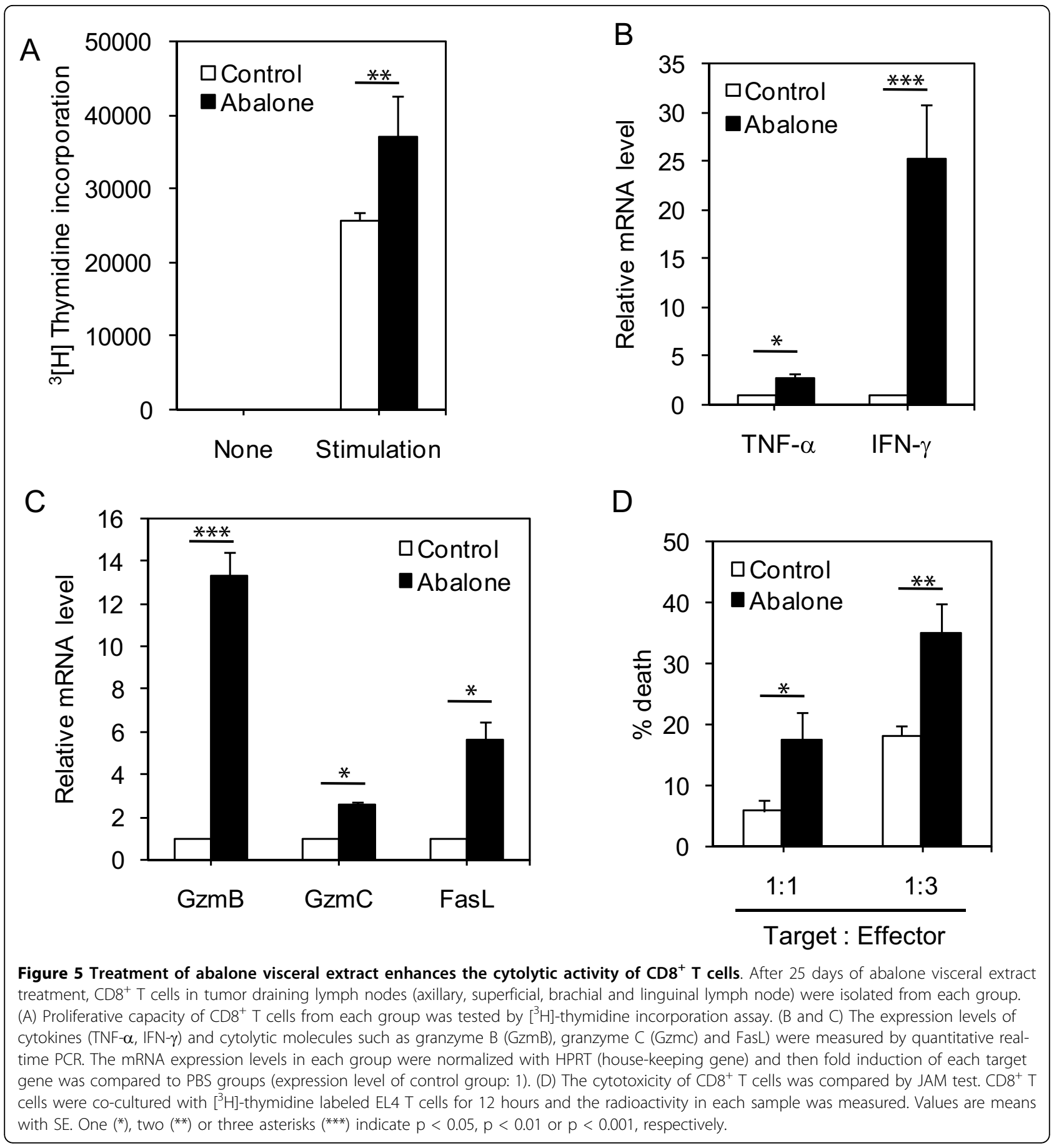

evidence and no detailed action mechanisms for its antitumor effects [7]. In the present study, we have demonstrated the potent anti-tumor efficacy of abalone visceral extract and have elucidated its underlying mechanism using a mouse breast cancer model that have high malignancy in tumor growth and metastasis [13,14]. Oral administration of abalone visceral extract significantly lowered tumor progression and metastasis by down-regulating the tumor-associated growth factors such as Cox-2, EGF, VEGF and FGF, while increasing the proliferation and cytolytic activity of $\mathrm{CD}^{+} \mathrm{T}$ cells.

Cyclooxygenase-2 (Cox-2) is an enzyme that catalyzes arachidonic acid to prostaglandins. Cox-2 is predominantly expressed in synoviocytes, fibroblasts, osteoblasts, activated endothelial cells and tumor cells [34,35]. Cox2 expression is induced by pro-inflammatory and 
mitogenic stimuli such as growth factors (EGF, FGF and VEGF) [36] and cytokines (TNF- $\alpha$ and IL1- $\beta$ ). Enhanced expression of Cox-2 is linked with tumor progression by inducing immune suppression as well as angiogenic and metastatic progression $[34,37,38]$. Elevated Cox-2 expression is associated with increased tumor size during breast cancer progression [22,39]. Modulation of Cox-2 expression by specific inhibitors is regarded as good chemopreventive approach for cancer treatment. However, Cox-2 inhibitors affect multiple cellular pathways and show some side effects [34,40]. Therefore, use of nutritive supplementation substances might be regarded as potential cancer preventive approach $[11,12]$. We have found that the oral administration of abalone visceral extract exerted anti-tumor growth effects by inhibiting tumor volume (up to $30 \%$ ) compared with control feeding group (Figure. 1). The mouse breast tumor induced by $4 \mathrm{~T} 1$ tumor cells mimics human breast cancer in the aspect of spontaneous metastasis to lung, lymph nodes, liver and bone [41]. Cox-2 expression has been identified as the marker for selective lung metastasis [27] in breast cancer model [20]. In this study, we used 4T1 mammary adenocarcinoma cells for tumor implantation. Oral administration of abalone visceral extract significantly inhibited tumor metastasis by modulating Cox-2 expression (Figure. 4). In accordance with our result, a previous study also demonstrated that treatment of either non-selective Cox inhibitor or selective Cox-2 inhibitor significantly reduced primary tumor growth and metastasis of $4 \mathrm{~T} 1$ breast cancer cells[42]. Even though reduction of Cox-2 expression does not exactly match with inhibition of Cox-2 activity by known inhibitors, specific knockdown of Cox-2 directly could reduce level of PGE2 synthesis in $4 \mathrm{~T} 1$ cells [23]. In line with aforementioned reports, we investigated whether abalone visceral extract changes Cox-2 expression upon treatment. Oral administration of abalone visceral extract reduced the metastatic splenomegaly (Figure. 2A and 2B) $[17,18]$ and lymphomegaly (data not shown) [43]. Metastatic breast cancer has a strong tendency to propagate into lung and bone $[19-21,26]$. Treatment of abalone visceral extract significantly inhibited lung metastasis (Figure. $2 \mathrm{C}$ and 2D) by decreasing Cox-2 expression level (Figure. 4A and 4B). Numerous evidences show that decreased level of Cox-2 is well correlated with metastatic inhibition from variety kinds of cancers [44]. Furthermore, previous data suggested that Cox-2 expression is associated with angiogenesis, lymph node metastasis, and apoptosis in human breast cancer [43] along with enhanced MMP-13 expression [28]. Interestingly, the expression levels of VEGF, EGF and MMP-13 are all decreased upon abalone visceral extract treatment (Figure. 4C). Collectively, oral administration of abalone visceral extract reduced metastatic progression by lowering Cox-2 expression and other target molecules including angiogenic factors and metalloproteinases in the metastatic tissues.

The tumor microenvironment induces active immune tolerance and escapes immune surveillance. Boosting the immune response can be one of the indirect ways to eliminate or suppress tumor growth via regulating immune homeostasis [30]. $\mathrm{CD}^{+} \mathrm{T}$ cells are known to have anti-tumor activity by killing the tumor antigens in an antigen specific or antigen non-specific way $[44,45]$. Tumor specific $\mathrm{CD}^{+} \mathrm{T}$ cells possess increased proliferation, cytolytic activity and induce expression of death related proteins and cytokines [31]. However, CD8 ${ }^{+}$ $\mathrm{T}$ cells at tumor sites or tumor draining lymph nodes frequently exhibit functional defects such as defective antigen specific cytolytic activity [46], lack of perforin expression [47], defective cytokine production and abnormal proliferation $[48,49]$. Enhanced $\mathrm{CD}^{+} \mathrm{T}$ cell activity is therefore critical to eradicate tumor cells, especially in tumor regions. In this study, oral administration of abalone visceral extract significantly inhibited tumor growth compared with the control (PBS-treated) group (Figure. 1 and Figure. 2). Administration of abalone visceral extract enhanced the cytolytic activity of $\mathrm{CD}^{+} \mathrm{T}$ cells by increasing the expression of effector molecules such as cytokines (IFN- $\gamma$ and TNF- $\alpha$ ) and cytolytic molecules (FasL, Gzm B and Gzm C) (Figure. $5 \mathrm{~B}$ and $5 \mathrm{C}$ ). Even though inflammatory cytokine signaling is the known stimulation for Cox-2 expression [24], increased expression of the cytokine in CD8 $+\mathrm{T}$ cells upon abalone visceral extract treatment can be explained by other mechanisms apart from Cox- 2 regulation by abalone visceral extract in tumor cells. In addition, abalone visceral extract significantly increased the specific lysis rate in the JAM test (Figure. 5D). Therefore, the enhanced effector function of $\mathrm{CD}^{+} \mathrm{T}$ cells by administration of abalone visceral extract may enhance anti-tumor immunity, which leads to suppression of tumor growth and metastasis to different organs.

\section{Conclusions}

Our data suggest that abalone visceral extract suppress primary tumor formation and inhibit tumor metastasis by attenuating the expression of Cox-2 and other target molecules including angiogenic factors and metalloproteinases. Furthermore, abalone visceral extract potentiate immune responses of $\mathrm{CD}^{+} \mathrm{T}$ cells by increasing their proliferation and cytolytic activity. Although further studies are needed to elucidate the exact active compounds responsible for the anti-tumor activity of abalone visceral extract, our data suggest the potential use of abalone visceral extract as an inhibitor of tumor growth and metastasis by targeting Cox-2 activity and the cytolytic effector function of $\mathrm{CD}^{+} \mathrm{T}$ cells. 


\section{Additional material}

Additional file 1: Supplementary Figure 1. Quantitative analysis of Laminarin and D-mannitol in abalone visceral extract using reverse phase high-performance liquid chromatography (RP-HPLC).

Additional file 2: Supplementary Figure 2. Toxicity test of the abalone visceral extract upon oral administration.

\section{List of abbreviations used}

COX-2: Cyclooxygenase-2; VEGF: Vascular endothelial growth factor; FGF: Fibroblast growth factor; EGF: Epidermal growth factor; MMP13: Matrix metallopeptidase 13; GZMB: Granzyme B; GZMC: Granzyme C; FASL: Fas Ligand; TNF- $\alpha$ : Tumor necrosis factor-alpha; IFN- $\gamma$ : Interferon-gamma.

\section{Acknowledgements}

This work was supported by grants from the BioGreen 21 Program (20070501034009; PJ007054) in Rural Development Administration and an Agricultural R\&D Promotion Center, Republic of Korea, RTI05-01-01 from the Regional Technology Innovation Program of the Ministry of Commerce, Industry and Energy, Korea Research Foundation funded by the Korean government (KRF-2007-313-C00507) and by the Systems Biology Infrastructure Establishment Grant provided by Gwangju Institute of Science and Technology.

\section{Author details}

${ }^{1}$ School of Life Sciences and Immune Synapse Research Center, Gwangju Institute of Science and Technology (GIST), Gwangju 500-712, Republic of Korea. ${ }^{2}$ Global leader program, Bugil Academy, Cheonan, Chungchengnamdo 330-941, Republic of Korea.

\section{Authors' contributions}

S-H.I and J.I.K designed the research; C-G.L, H-K.K and C-R.I conducted research; J.H.R and S.J.K helped the HPLC experiments and analysis; C-G.L and S-H.I analyzed data; C-G.L and S-H.I wrote the paper; S-H.I had primary responsibility for final content. All authors read and approved the final manuscript.

\section{Competing interests}

The authors declare that they have no competing interests.

Received: 19 April 2010 Accepted: 20 October 2010 Published: 20 October 2010

\section{References}

1. Wikipedia contributors:[http://en.wikipedia.org/w/index.php? title=Abalone\&oldid=306576568], Abalone [Internet]. Wikipedia TFEA, [cited 2009 Aug 7].

2. Hwang E, Baek J, Park C: The mass cultivation of Ecklonia stolonifera Okamura as a summer feed for the abalone industry in Korea. Journal of Applied Phycology 2009.

3. Smit AJ: Medicinal and pharmaceutical uses of seaweed natura products: A review. Journal of Applied Phycology 2004, 16(4):245-262.

4. Zhu B-W, Wang L-S, Zhou D-Y, Li D-M, Sun L-M, Yang J-F, Wu H-T, Zhou XQ, Tada M: Antioxidant activity of sulphated polysaccharide conjugates from abalone (Haliotis discus hannai Ino). European Food Research and Technology 2008, 227(6):1663-1668.

5. Kim HL, Kang SG, Kim IC, Kim SJ, Kim DW, Ma SJ, Gao T, Li H, Kim MJ, Lee $\mathrm{TH}$, et al: In vitro Anti-hypertensive, Antioxidant and Anticoagulant Activities of Extracts from Haliotis discus hannai. Journal of The Korean Society of Food Science and Nutrition 2006, 35(7):835-840, Korean.

6. Khan N, Afaq F, Mukhtar H: Cancer Chemoprevention Through Dietary Antioxidants: Progress and Promise. Antioxidants \& Redox Signaling 2008, 10(3):475-510

7. Uchida H, Sasaki T, Uchida NA, Takasuka N, Endo Y, Kamiya H: Oncostatic and immunomodulatory effects of a glycoprotein fraction from water extract of abalone, Haliotis discus hannai. Cancer Immunology, Immunotherapy 1987, 24(3):207-212.
8. Jemal A, Siegel R, Ward E, Hao Y, Xu J, Murray T, Thun MJ: Cancer Statistics, 2008. CA Cancer J Clin 2008, 58(2):71-96.

9. Greenberg PA, Hortobagyi GN, Smith TL, Ziegler LD, Frye DK, Buzdar AU: Long-term follow-up of patients with complete remission following combination chemotherapy for metastatic breast cancer. J Clin Oncol 1996, 14(8):2197-2205.

10. Slamon DJ, Leyland-Jones B, Shak S, Fuchs H, Paton V, Bajamonde A Fleming T, Eiermann W, Wolter J, Pegram M, et al: Use of Chemotherapy plus a Monoclonal Antibody against HER2 for Metastatic Breast Cancer That Overexpresses HER2. N Engl J Med 2001, 344(11):783-792.

11. Gonzalez CA: Nutrition and cancer: the current epidemiological evidence. British Journal of Nutrition 2006, 96(S1):S42-S45

12. Donaldson M: Nutrition and cancer: $A$ review of the evidence for an anticancer diet. Nutrition Journal 2004, 3(1):19.

13. Huang X, Wong MK, Yi H, Watkins S, Laird AD, Wolf SF, Gorelik E: Combined Therapy of Local and Metastatic 4T1 Breast Tumor in Mice Using SU6668, an Inhibitor of Angiogenic Receptor Tyrosine Kinases, and the Immunostimulator B7.2-IgG Fusion Protein. Cancer Res 2002, 62(20):5727-5735.

14. Pulaski BA, Ostrand-Rosenberg S: Reduction of Established Spontaneous Mammary Carcinoma Metastases following Immunotherapy with Major Histocompatibility Complex Class II and B7.1 Cell-based Tumor Vaccines. Cancer Res 1998, 58(7):1486-1493.

15. Ho-Keun K, Won Kyung J, Ji-Sun H, Choong-Gu L, Jae-Seon S, Jin AP, Byoung Seob K, Sin-Hyeog I: Cinnamon extract suppresses tumor progression by modulating angiogenesis and the effector function of CD8+ T cells. Cancer letters 2009, 278(2):174-182.

16. Usharauli D, Perez-Diez A, Matzinger P: The JAM Test and its daughter PJAM: simple tests of DNA fragmentation to measure cell death and stasis. Nat Protocols 2006, 1(2):672-682.

17. Baruch K, Moshe S, Abraham K, Marianne S, Dany B, Eliezer R, David G: Splenomegaly and solitary spleen metastasis in solid tumors. Cancer 1987, 60(1):100-102.

18. duPre SA, Hunter JKW: Murine mammary carcinoma 4T1 induces a leukemoid reaction with splenomegaly: Association with tumor-derived growth factors. Experimental and Molecular Pathology 2007, 82(1):12-24.

19. Muller A, Homey B, Soto H, Ge N, Catron D, Buchanan ME, McClanahan T, Murphy E, Yuan W, Wagner SN, et al: Involvement of chemokine receptors in breast cancer metastasis. Nature 2001, 410(6824):50-56.

20. Gupta GP, Nguyen DX, Chiang AC, Bos PD, Kim JY, Nadal C, Gomis RR, Manova-Todorova K, Massague J: Mediators of vascular remodelling coopted for sequential steps in lung metastasis. Nature 2007, 446(7137):765-770.

21. Olkhanud PB, Baatar D, Bodogai M, Hakim F, Gress R, Anderson RL, Deng J, Xu M, Briest S, Biragyn A: Breast Cancer Lung Metastasis Requires Expression of Chemokine Receptor CCR4 and Regulatory T Cells. Cancer Res 2009, 69(14):5996-6004.

22. Ristimaki A, Sivula A, Lundin J, Lundin M, Salminen T, Haglund C, Joensuu H, Isola J: Prognostic Significance of Elevated Cyclooxygenase-2 Expression in Breast Cancer. Cancer Res 2002, 62(3):632-635.

23. Neil JR, Johnson KM, Nemenoff RA, Schiemann WP: Cox-2 inactivates Smad signaling and enhances EMT stimulated by TGF-beta through a PGE2-dependent mechanisms. Carcinogenesis 2008, 29(11):2227-2235.

24. Ghosh N, Chaki R, Mandal V, Mandal SC: COX-2 as a target for cance chemotherapy. Pharmacol Rep 2010, 62(2):233-244.

25. Bergers G, Benjamin LE: Tumorigenesis and the angiogenic switch. Nat Rev Cancer 2003, 3(6):401-410.

26. Singh B, Berry JA, Shoher A, Ayers GD, Wei C, Lucci A: COX-2 involvement in breast cancer metastasis to bone. Oncogene 2007, 26(26):3789-3796.

27. Minn AJ, Gupta GP, Siegel PM, Bos PD, Shu W, Giri DD, Viale A, Olshen AB, Gerald WL, Massague J: Genes that mediate breast cancer metastasis to lung. Nature 2005, 436(7050):518-524.

28. Zhang B, Cao X, Liu Y, Cao W, Zhang F, Zhang S, Li H, Ning L, Fu L, Niu Y, et al: Tumor-derived matrix metalloproteinase-13 (MMP-13) correlates with poor prognosis of invasive breast cancer. BMC Cancer 2008, 8(1):83.

29. Larkins T, Nowell M, Singh S, Sanford G: Inhibition of cyclooxygenase-2 decreases breast cancer cell motility, invasion and matrix metalloproteinase expression. BMC Cancer 2006, 6(1):181.

30. Lake RA, Robinson BWS: Immunotherapy and chemotherapy - a practical partnership. Nat Rev Cancer 2005, 5(5):397-405. 
31. Trapani JA, Smyth MJ: Functional significance of the perforin/granzyme cell death pathway. Nat Rev Immunol 2002, 2(10):735-747.

32. Dranoff G: Cytokines in cancer pathogenesis and cancer therapy. Nat Rev Cancer 2004, 4(1):11-22.

33. Glimcher LH, Townsend MJ, Sullivan BM, Lord GM: Recent developments in the transcriptional regulation of cytolytic effector cells. Nat Rev Immunol 2004, 4(11):900-911.

34. Gasparini G, Longo R, Sarmiento R, Morabito A: Inhibitors of cyclooxygenase 2: a new class of anticancer agents? The Lancet Oncology 2003, 4(10):605-615.

35. Tomozawa S, Tsuno NH, Sunami E, Hatano K, Kitayama J, Osada T, Saito S, Tsuruo T, Shibata Y, Nagawa H: Cyclooxygenase-2 overexpression correlates with tumour recurrence, especially haematogenous metastasis, of colorectal cancer. British Journal of Cancer 2000, 83(3):324-328.

36. Schneider BP, Miller KD: Angiogenesis of Breast Cancer. J Clin Oncol 2005, 23(8):1782-1790.

37. Leahy KM, Ornberg RL, Wang Y, Zweifel BS, Koki AT, Masferrer UL: Cyclooxygenase-2 Inhibition by Celecoxib Reduces Proliferation and Induces Apoptosis in Angiogenic Endothelial Cells in Vivo. Cancer Res 2002, 62(3):625-631.

38. Zou W: Immunosuppressive networks in the tumour environment and their therapeutic relevance. Nat Rev Cancer 2005, 5(4):263-274.

39. Wülfing P, Diallo R, Müller C, Wülfing C, Poremba C, Heinecke A, Rody A, Greb RR, Böcker W, Kiesel L: Analysis of cyclooxygenase-2 expression in human breast cancer: high throughput tissue microarray analysis. Journal of Cancer Research and Clinical Oncology 2003, 129(7):375-382.

40. Wang D, DuBois RN: PROSTAGLANDINS AND CANCER. Gut 2006, 55(1):115-122.

41. Lelekakis M, Moseley JM, Martin TJ, Hards D, Williams E, Ho P, Lowen D, Javni J, Miller FR, Slavin J, et al: A novel orthotopic model of breast cancer metastasis to bone. Clinical and Experimental Metastasis 1999, 17(2):163-170

42. Connolly EM, Harmey JH, O'Grady T, Foley D, Roche-Nagle G, Kay E, Bouchier-Hayes DJ: Cyclo-oxygenase inhibition reduces tumour growth and metastasis in an orthotopic model of breast cancer. $\mathrm{Br} J$ Cancer 2002, 87(2):231-237.

43. Costa C, Soares R, Reis-Filho JS, Leitao D, Amendoeira I, Schmitt FC: Cyclooxygenase 2 expression is associated with angiogenesis and lymph node metastasis in human breast cancer. J Clin Pathol 2002, 55(6):429-434.

44. Greenhough A, Smartt HJM, Moore AE, Roberts HR, Williams AC Paraskeva C, Kaidi A: The COX-2/PGE2 pathway: key roles in the hallmarks of cancer and adaptation to the tumour microenvironment. Carcinogenesis 2009, 30(3):377-386.

45. Pittet MJ: Behavior of immune players in the tumor microenvironment. Current Opinion in Oncology 2009, 21(1):53-59.

46. Zippelius A, Batard P, Rubio-Godoy V, Bioley G, Lienard D, Lejeune F, Rimoldi D, Guillaume P, Meidenbauer N, Mackensen A, et al: Effector Function of Human Tumor-Specific CD8 T Cells in Melanoma Lesions: A State of Local Functional Tolerance. Cancer Res 2004, 64(8):2865-2873.

47. Radoja S, Saio M, Schaer D, Koneru M, Vukmanovic S, Frey AB: CD8+ Tumor-Infiltrating T Cells Are Deficient in Perforin-Mediated Cytolytic Activity Due to Defective Microtubule-Organizing Center Mobilization and Lytic Granule Exocytosis. J Immunol 2001, 167(9):5042-5051.

48. Van Den Hove LE, Van Gool SW, Van Poppel H, Baert L, Coorevits L, Van Damme B, Ceuppens JL: Phenotype, cytokine production and cytolytic capacity of fresh (uncultured) tumour-infiltrating $T$ lymphocytes in human renal cell carcinoma. Clinical \& Experimental Immunology 1997, 109(3):501-509.

49. Nakagomi H: Lack of interleukin-2 (IL-2) expression and selective expression of IL-10 mRNA in human renal cell carcinoma. International Journal of Cancer 1995, 63(3):366-371.

Pre-publication history

The pre-publication history for this paper can be accessed here: http://www.biomedcentral.com/1472-6882/10/60/prepub

doi:10.1186/1472-6882-10-60

Cite this article as: Lee et al: Abalone visceral extract inhibit tumor growth and metastasis by modulating Cox-2 levels and CD8+ T cell activity. BMC Complementary and Alternative Medicine 2010 10:60.

\section{Submit your next manuscript to BioMed Central and take full advantage of:}

- Convenient online submission

- Thorough peer review

- No space constraints or color figure charges

- Immediate publication on acceptance

- Inclusion in PubMed, CAS, Scopus and Google Scholar

- Research which is freely available for redistribution

Submit your manuscript at www.biomedcentral.com/submit 